\title{
The many aspects of quantum dots
}

\author{
From fundamental physics and chemistry to digital cameras, improved displays and more natural \\ lighting, nanoscale semiconductor structures called quantum dots are having an impact on many \\ areas of science and technology.
}

For some reason, semiconductor quantum dots do not feature prominently in the history of nanotechnology. Like molecular beam epitaxy ${ }^{1}$, for instance, they have had a much lower profile than the carbon nanotube and various forms of scanning probe microscopy ${ }^{2}$. However, quantum dots have been around for at least as long as either of these stalwarts of nanotech, and have been the focus of just as much research. And although they might lack the pleasing natural geometry that is found in nanotubes (and also in fullerenes and graphene), the fact that quantum dots can be made from many different elements, and come in many different shapes and sizes, gives them undoubted advantages over higher-profile carbonbased nanomaterials for some uses. This is reflected by the range of basic science that can be studied with quantum dots ${ }^{3}$, and the breadth of potential applications for these materials - and disputes about patents.

\section{Colloidal quantum dots emerged from a number of research labs in the early 1980s.}

Broadly speaking, there are two families of quantum dots: chemists prefer to use 'wet' methods to produce relatively large volumes of colloidal quantum dots, which are usually passivated by organic molecules on their surface; physicists, on the other hand, tend to work with epitaxial quantum dots that are painstakingly defined by electrostatic gates on substrates, or that self-assemble on surfaces. It is also possible to make a quantum dot by trapping a single molecule or nanoparticle between two electrodes, by attaching electrodes to a nanotube or to graphene, or by modulating the level of doping in a single crystal of a semiconductor ${ }^{4}$. Moreover, further variety is possible because the nanoparticles trapped between the electrodes can, for example, be metallic, ferromagnetic or superconducting ${ }^{5}$
Essential characteristics of quantum dots include the fact that the energy levels occupied by the charge carriers are quantized, as in atoms and molecules, and that the bandgap between the conduction and valence bands increases as the dot gets smaller, which decreases the wavelength at which they fluoresce. Electron-electron interactions also become stronger, single-electron effects such as the Coulomb blockade are observed, and chemical properties such as the redox potential change ${ }^{6}$.

Colloidal quantum dots emerged from a number of research labs in the early 1980s, with Louis Brus (then at Bell Labs, now at Columbia University) being singled out to receive the first Kavli Prize in Nanoscience in 2008 for his pioneering efforts in this field ${ }^{7,8}$. They have been widely used in bio-imaging applications for a number of years, and researchers have recently developed methods to stop the fluorescence from quantum dots switching on and off in a random manner (a problem known as 'blinking') ${ }^{9}$. More recently the optical properties of colloidal quantum dots have been exploited in lighting to improve energy efficiency and make the output of light-emitting diodes seem more natural ${ }^{10}$, and applications in digital cameras, displays and solar power are also being investigated ${ }^{10,11}$. A challenge facing all companies building products with colloidal quantum dots is to move away from toxic materials such as cadmium.

Research into epitaxial quantum dots was a natural progression of work that began with studies of two-dimensional electron gases in multilayer semiconductor devices, continued with experiments on quantum wires, and eventually arrived at zero-dimensional nanostructures in the late 1980s. Mark Reed (then at Texas Instruments, now at Yale University) is widely credited with coining the term 'quantum dots' in a paper ${ }^{12}$ published in 1988

Two articles in this issue illustrate the ongoing appeal of quantum dots from the perspectives of applications and fundamental research. On page 391, Gerasimos Konstantatos and Edward Sargent review the use of colloidal quantum dots and metal nanoparticles for applications in photon detection. Making competitive detectors involves optimizing a number of factors including the absorption, response time and noise.

\section{A challenge is to move away from toxic materials such as cadmium.}

On page 458, Silvano de Franceschi and co-workers describe experiments with selfassembled silicon-germanium quantum dots on a silicon substrate. Improved understanding of the behaviour of charge carriers (holes, in this instance) in such quantum-dot structures is necessary for the development of new devices for applications in electronics and spintronics. The development of single-photon sources is another area of intense activity among physicists working in the quantumdot community ${ }^{3}$.

But no matter how they are made or what they do, quantum dots look set to figure more prominently in the future of nanotechnology than they have in its history.

\footnotetext{
References

1. McCray, W. P. Nature Nanotech. 2, 259-261 (2007).

2. Toumey, C. Nature Nanotech. 5, 239-241 (2010).

3. Quantum Dot 2010 (IOP, Nottingham, 2010); www.qd2010.org/activity/overview/page_34302.html

4. Fuechsle, M. et al. Nature Nanotech. doi:10.1038/ nnano.2010.95 (2010).

5. Hanson, R., Kouwenhoven, L. P., Petta, J. R., Tarucha, S. \& Vandersypen, L. M. K. Rev. Mod. Phys. 79, 1217-1265 (2007).

6. Brus, L. E. Nano Lett. 10, 363-365 (2010).

7. Rossetti, R., Beck, B. M. \& Brus, L. E. J. Chem. Phys. 79, 1086-1088 (1983).

8. Brus, L. E. Kavli Prize Lecture: Semiconductor Nanocrystals; available via http://go.nature.com/jI95ft

9. Wang, X. et al. Nature 459, 686-689 (2000).

10. The Economist: Technology Quarterly 15-16 (4 May, 2010); available via http://go.nature.com/kXQFCf

11. Sanderson, K. Nature 459, 760-761 (2009).

12. Reed, M. A. et al. Phys. Rev. Lett. 60, 535-537 (1988).
} 